\title{
BMJ Open Imaging predictors of poststroke depression: methodological factors in voxel-based analysis
}

\author{
Sophia A Gozzi, ${ }^{1,2}$ Amanda G Wood, ${ }^{2,3}$ Jian Chen, ${ }^{2,4}$ Krishnarao Vaddadi, ${ }^{1,5}$ \\ Thanh G Phan ${ }^{2}$
}

To cite: Gozzi SA, Wood AG, Chen J, et al. Imaging predictors of poststroke depression: methodological factors in voxel-based analysis. BMJ Open 2014;4: e004948. doi:10.1136/ bmjopen-2014-004948

- Prepublication history for this paper is available online. To view these files please visit the journal online (http://dx.doi.org/10.1136/ bmjopen-2014-004948).

Received 28 January 2014 Revised 22 May 2014 Accepted 30 May 2014

\section{CrossMark}

\author{
1Department of Medicine, \\ School of Psychology and \\ Psychiatry, Monash \\ University, Melbourne, \\ Australia \\ 2Department of Medicine, \\ Stroke and Ageing Research \\ Group, Southern Clinical \\ School, Monash University, \\ Melbourne, Australia \\ ${ }^{3}$ School of Psychology, \\ University of Birmingham, \\ Edgbaston, UK \\ ${ }^{4}$ Department of \\ Developmental Imaging, \\ Murdoch Children's Research \\ Institute, Melbourne, \\ Australia \\ ${ }^{5}$ Southern Health, Melbourne, \\ Australia
}

Correspondence to Dr Amanda G Wood; a.g.wood@bham.ac.uk

\section{ABSTRACT}

Objective: The purpose of this study was to explore the relationship between lesion location and poststroke depression using statistical parametric mapping.

Methods: First episode patients with stroke were assessed within 12 days and at 1-month poststroke. Patients with an a priori defined cut-off score of 11 on the Hospital Anxiety and Depression Scale (HADS) at follow-up were further assessed using the MiniInternational Neuropsychiatric Interview (MINI) to confirm a clinical diagnosis of major or minor depression in accordance with Diagnostic and Statistical Manual-IV (DSM-IV) inclusion criteria. Participants were included if they were aged 1885 years, proficient in English and eligible for MRI. Patients were excluded if they had a confounding diagnosis such as major depressive disorder at the time of admission, a neurodegenerative disease, epilepsy or an imminently life-threatening comorbid illness, subarachnoid or subdural stroke, a second episode of stroke before follow-up and/or a serious impairment of consciousness or language. Infarcts observed on MRI scans were manually segmented into binary images, linearly registered into a common stereotaxic coordinate space. Using statistical parametric mapping, we compared infarct patterns in patients with stroke with and without depression.

Results: 27\% (15/55 patients) met criteria for depression at follow-up. Mean infarct volume was 19 $\pm 53 \mathrm{~mL}$ and National Institute of Health Stroke Scale (NIHSS) at Time 1 (within 12 days of stroke) was $4 \pm 4$, indicating a sample of mild strokes. No voxels or clusters were significant after a multiple comparison correction was applied $(p>0.05)$. Examination of infarct maps showed that there was minimal overlap of infarct location between patients, thus invalidating the voxel comparison analysis.

Conclusions: This study provided inconclusive evidence for the association between infarcts in a specific region and poststroke depression.

\section{INTRODUCTION}

Poststroke depression (PSD) has been reported to affect more than $30 \%$ of patients with stroke. It has been associated with

\section{Strengths and limitations of this study}

- This prospective study is the first lesion location study in poststroke depression to use voxelbased analysis. It demonstrates the challenges in using this method of analysis for this cohort and discusses ways of addressing these issues in future research.

- Limitations include that the study provided inconclusive evidence for the association between infarcts in a specific region and poststroke depression.

- Infarct volumes were small, precluding further analysis and suggested that we had unintentionally recruited patients with mild strokes.

increased mortality, ${ }^{1-3}$ cognitive impairment, ${ }^{1}$ greater functional impairments, ${ }^{4}$ poorer rehabilitation outcomes ${ }^{2}{ }_{3}$ and reduced health-related quality of life. ${ }^{5}$ An understanding of patients at risk of PSD would help guide preventive interventions. To date there is consensus that stroke severity, cognitive impairment, physical disability and handicap correlate with PSD. ${ }^{6}$ The postulate for a neurobiological basis of PSD was based on observed behavioural changes in rats following focal cortical lesions, and concurrent change in catecholamine levels. ${ }^{7}$ Human studies later suggested that depression was more likely after stroke affecting the left hemisphere or frontal lobe. ${ }^{8}$ This idea of lesion location impacting on PSD is an attractive one given the finding of lesion location causing neurological deficit poststroke. ${ }^{9}$ However, the role of lesion location in PSD remains a point of controversy due to conflicting results, ${ }^{6} \quad 10 \quad 11$ which could be related to methodological differences between studies.

Modern studies of lesion location use brain imaging analysis tools to characterise ensembles of voxels representing the network of regions involved. ${ }^{9}$ However, earlier studies 
and those assessed in review papers focused on coarse analyses differentiating left from right hemisphere stroke or anterior versus posterior lesion locations, and their resultant relationship with depression. In light of the improved sensitivity to regional abnormalities afforded by voxel-based analysis, the aim of this study was to examine the role of lesion location in PSD. It was hypothesised that there would be a relationship between lesion location and PSD. In the process of performing this analysis we encountered several issues with voxelbased analysis for depression in patients with stroke.

\section{MATERIALS AND METHODS \\ Participants}

Participants comprised patients who presented to Monash Medical Centre and Dandenong Hospital with a first cerebral infarction or haemorrhage $(\mathrm{N}=71)$ in Melbourne, Australia between May 2009 and September 2010. In total, 717 patients were diagnosed with ischaemic stroke and 247 patients with intracerebral haemorrhage during this time frame. Participants were included if they were aged 18-85 years, proficient in English (ie, capable of completing the assessment materials), and eligible for MRI. Participants were excluded if they had a compounding diagnosis (major depressive disorder at the time of admission, a neurodegenerative disease, epilepsy or an imminently life-threatening comorbid illness), had a subarachnoid or subdural haemhorrage, if a second episode of stroke occurred before follow-up, and/or they were deemed incapable of participation due to incapacity (serious impairment of consciousness or language). The language component of the National Institute of Health Stroke Scale (NIHSS) and the Multilingual Aphasia Examination's (MAE) Token Test (Form A), a 22-item test of oral language comprehension, ${ }^{12}$ were used to complement clinical judgment in cases with language disturbance. Ethics approval was received from Southern Health and Monash University Human Research Ethics Committees. All participants provided written informed consent.

\section{Test administration and procedure}

MRI scans were performed as part of clinical care. Cognitive functioning, stroke severity, physical disability and handicap and demographic information were acquired at Time 1 (typically within 10 days of stroke). Depression was assessed at Time 2 (1 month since stroke).

\section{Measures}

Cognitive functioning was assessed using the Addenbrooke's Cognitive Examination Revised (ACE-R). ${ }^{13}$ The NIHSS was used to determine stroke severity. ${ }^{14}$ The Modified Rankin Scale (MRS) was used to measure physical disability and handicap. ${ }^{15} 16$ The scale ranges from 0 to 6 from 'No symptoms at all', to 'Death'. The Hospital Anxiety and Depression Scale (HADS) has been used routinely within stroke to screen for symptoms of depression. ${ }^{17}$ Those with a cut-off score of 11 on the HADS were further assessed using the Mini-International Neuropsychiatric Interview (MINI), a semistructured clinical interview, to confirm a clinical diagnosis of mood disorder due to stroke with major depressive-like episode or with depressive features in accordance with Diagnostic and Statistical Manual-IV (DSM-IV) inclusion criteria, ${ }^{18} 19$ abbreviated as 'major' and 'minor' depression below. The MINI was administered by a provisional psychologist. This procedure was used to optimise sensitivity, as a cut-off score of 11 on the HADS has been found to have a sensitivity of 86.8 for detecting major and minor depression, and a specificity of $69.9 .^{20}$ The MINI displays high validity and reliability in relation to the Structured Clinical Interview for DSM, Patient Edition (SCID-P), and the Composite International Diagnostic Interview (CIDI). The MINI can be administered in a shorter period of time than the aforementioned tools. ${ }^{18}$ A history of depression was documented if noted in the medical file or reported during the clinical interview.

\section{Image acquisition}

MRI scans were performed on a $1.5 \mathrm{~T}$ superconducting imaging system (General Electric Medical Systems, Milwaukee, Wisconsin, USA) with echoplanar imaging capabilities. T2 images were acquired using thickness 6/1.7 mm, matrix $256 \times 256$ and TR/TE/ETL 2 000/ 102/12. Diffusion-weighted imaging (DWI) was performed with $6 / 1.7 \mathrm{~mm}$ thickness, matrix $128 \times 256$, field of view $230 \mathrm{~mm}$ and TR/TE $10000 / 102$. Diffusion gradient values of 0 and $1000 \mathrm{~s} / \mathrm{mm}^{2}$ were applied in three directions. Isotropic apparent diffusion coefficient maps were calculated on a voxel-by-voxel basis. The threedimensional time-of-flight MR angiogram was performed using TR/TE $38 / 6.9,25^{\circ}$ flip angle, thickness $1.4 \mathrm{~mm}$, slab thickness $60 \mathrm{~mm}$, matrix $256 \times 224$ and field of view $180 \mathrm{~mm}$.

\section{Registration and segmentation}

Alignment of corresponding anatomical structures in images (prior to segmentation) from different participants was achieved by linear registration to a standard brain template comprising images from 152 participants placed into the stereotaxic coordinate space (MNI template available at http://www.bic.mni.mcgill.ca/software/). Infarcts were manually segmented on the native $\mathrm{T}_{2}$-weighted images using interactive Display, mousedriven software and standardised intensity windows. The transformation matrix was used to convert native segmented images into standard stereotaxic space.

\section{t Statistics}

A parametric voxel-based analysis (SPM; Wellcome Trust Centre for Neuroimaging, London, England) was used to produce statistical parametric maps. Images were spatially smoothed with a Gaussian kernel of $12 \mathrm{~mm}$. This analysis used the two-sample t test in SPM5 to compare 
the distribution of the means of infarcts of patients with and without depression. A spatial mask of the sum of all infarcted regions in this sample (based on images before they were spatially smoothed) was applied so that the voxel analysis was constrained to this area. A false discovery rate (FDR) was used to correct for multiple comparisons, as the t statistic is applied to many voxels within the images. The FDR controls the expected proportion of false positives among the voxels that have exceeded a certain threshold on the raw $t$ statistics map. $^{21}$ The FDR was set at 0.05 so that among the significant voxels above the threshold, $5 \%$ would be considered false positive. To assess the validity of the t-statistics images, an average map of the infarcts was generated. This process allows determination if the analysis contains infarcts which cover most of the brain. Previous investigators have been criticised for performing studies on infarcts belonging to a restricted arterial territory and hence in this study we include infarcts from any arterial territory. ${ }^{22}$

\section{Volume and overlap of infarcts}

The volume of each infarct was calculated using a voxel counting algorithm. The overlap of infarcts among patients was manually examined to aid interpretation of results. The maximum overlap of regions of infarction between (1) all patients was seven; (2) depressed patients was two; and (3) patients without depression was seven (see figures 1 and 2). A mask was used to determine the volume of infarcts in the anterior and posterior regions of the left and right hemispheres.

\section{RESULTS}

\section{Clinical characteristics}

Of the 71 participants, 55 (31 males) completed all aspects of the study including an MRI scan. Ten of the 71 patients withdrew or were lost to follow-up, five patients did not undergo a clinical MRI scan and one was excluded following a second episode of stroke. The clinical characteristics of participants are summarised in table 1 . The mean age was 62.9 years $(\mathrm{SD}=13.6)$. Eighty per cent $(n=44)$ of patients had ischaemic strokes. Twelve patients $(22 \%)$ had a personal history of depression. The mean NIHSS at Time 1 was $4 \pm 4$, representing minor strokes. Acute and follow-up assessments were administered within an average of $6 \pm 3$ days (range 1-12) and $33 \pm 8$ (range 25-74) days of stroke, respectively. MRI scans were performed within an average of 20.8 \pm 23.7 days of stroke (0-85 days). Overall, $27.3 \%$ of patients experienced depression $(n=15)$, either major $(\mathrm{n}=9 ; 16.4 \%)$ or minor $(\mathrm{n}=6 ; 10.9 \%)$. The mean total HADS score was $10 \pm 7(0-29)$. Three patients $(6 \%)$ were taking antidepressant medication during the course of the study.

\section{Image analysis}

The mean infarct volume was $19.4 \pm 53.2 \mathrm{~mL} \quad(0-$ $349.8 \mathrm{~mL})$. The mean volume of haemorrhagic lesions was $44.7 \pm 103.9(0.12-349.83 \mathrm{~mL})$. Stroke occurred in the left hemisphere in 29 patients $(52.7 \%)$, right hemisphere in 20 patients $(36.4 \%)$. It was present in both hemispheres in five patients $(9.1 \%)$. The mean volume of infarct in the left hemisphere was $6.2 \pm 15.6 \mathrm{~mL}$ and the right hemisphere was $38.0 \pm 83.2 \mathrm{~mL}(\mathrm{p}=0.001)$. The

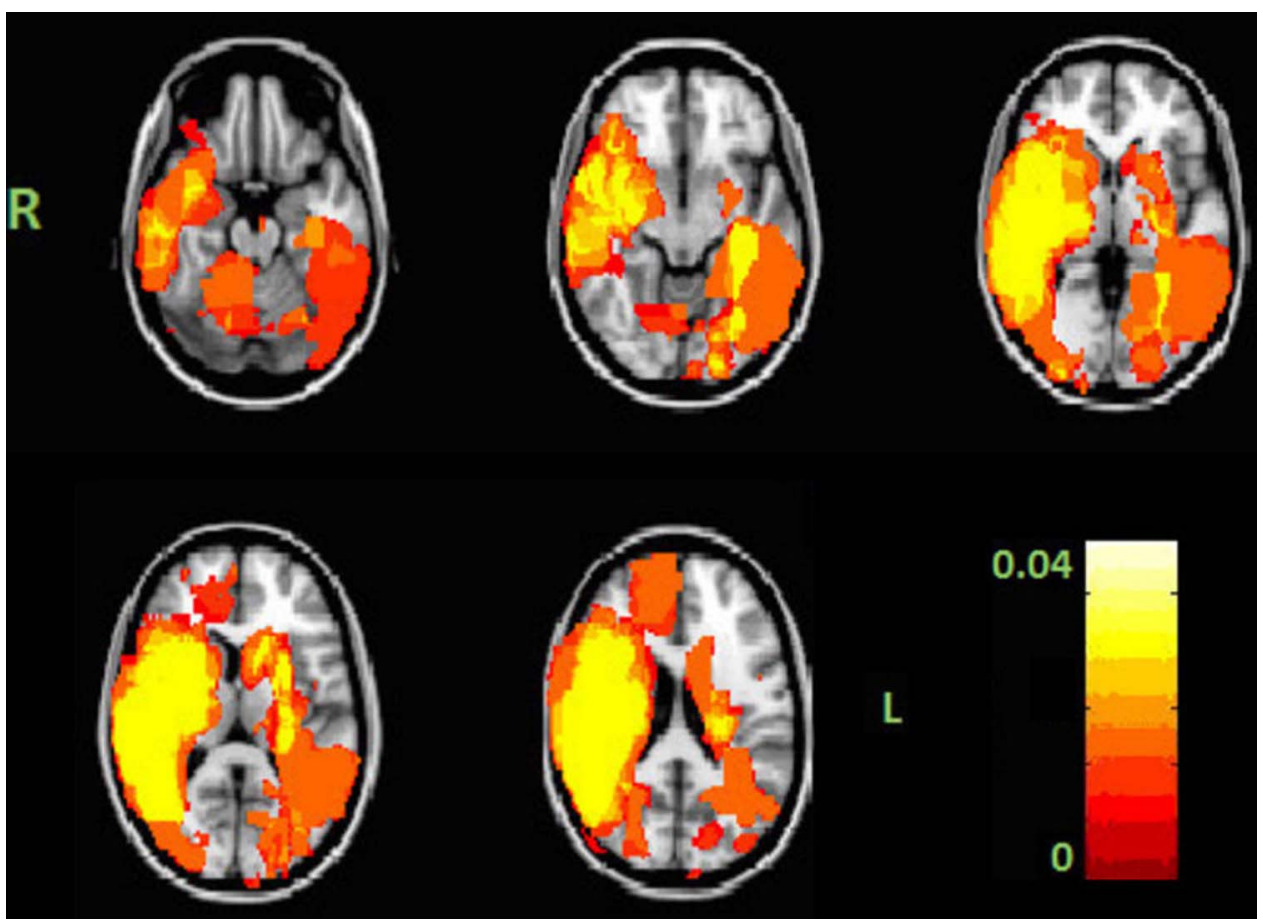

Figure 1 Average map of infarcts for all patients in stereotaxic coordinate space from $z=-20$ to 20 (from left to right). 


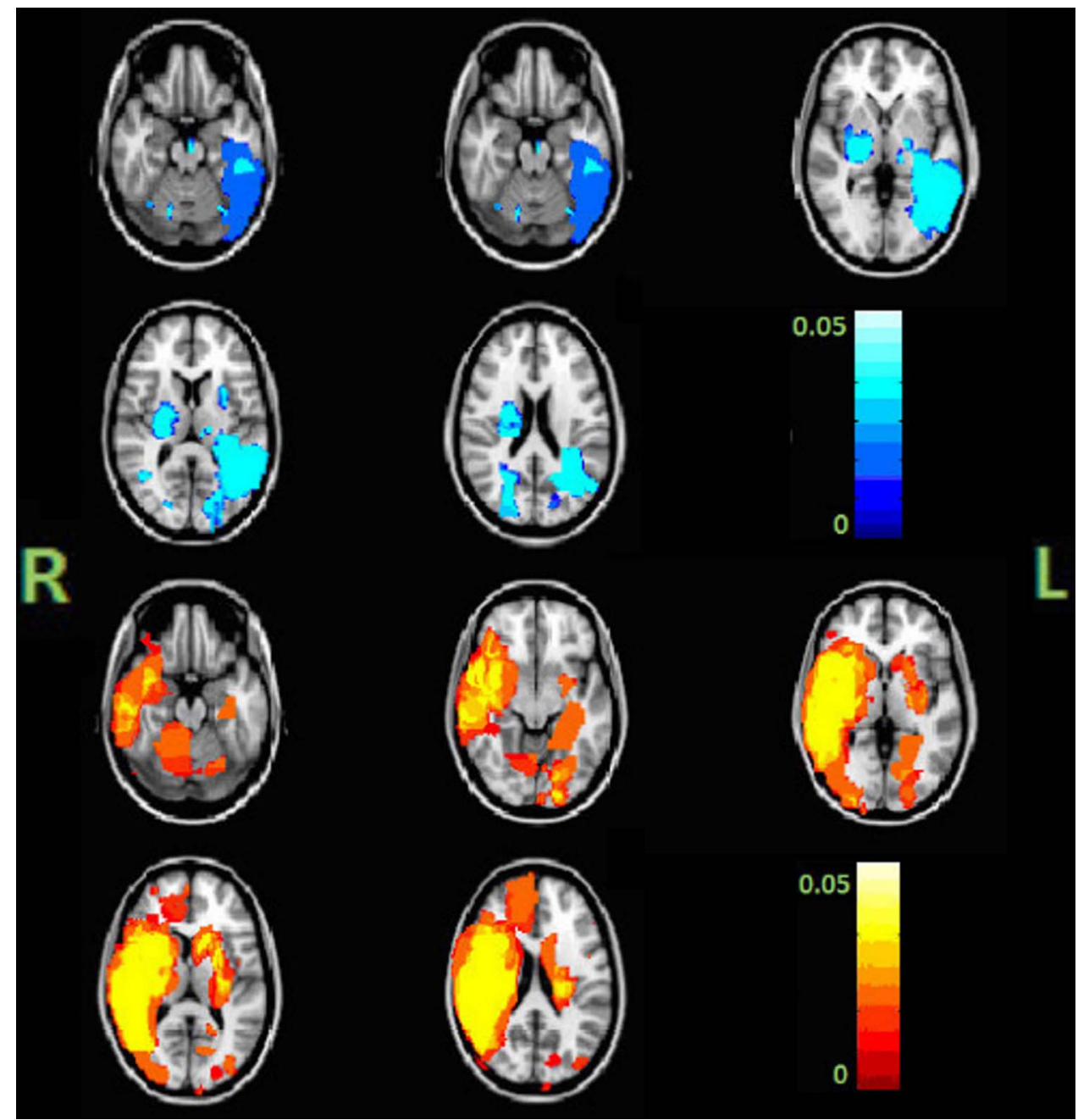

Figure 2 Average map of infarcts for the depressed (blue) and not depressed (yellow) groups in stereotaxic coordinate space from $z=-20$ to 20 (from left to right).

mean volume of infarct in the left anterior region was $4.8 \pm 7.7 \mathrm{~mL}$ and the right anterior region was 46.9 $\pm 91.4 \mathrm{~mL}(\mathrm{p}=0.06)$. The mean volume of infarct in the left posterior region was $16.2 \pm 27.2$ compared with 17.6 $\pm 19.8 \mathrm{~mL}$ for the right posterior region $(\mathrm{p}>0.05)$. Modified Rankin Scale scores greater than 2 were associated with larger lesion volumes $(\mathrm{p}=0.02)$ and there was a large and medium correlation respectively between NIHSS scores acutely and at 1 month and lesion volumes $(\mathrm{p}<0.05)$.

The average map of infarcts is displayed in figure 1 and showed that infarcts occurred predominantly around the internal capsule and striatocapsular region. Infarct extent was greater on the right. The infarct pattern of the depressed group versus the group without depression was significant at the uncorrected level $(p<0.01$, see figure 2$)$. No voxels or clusters were significant after the FDR correction was applied $(\mathrm{p}>0.05)$. Additionally, there was no relationship between left anterior strokes and depression $(\mathrm{p}>0.05)$. Infarct location of stroke by group is displayed in table 2 .
When potential confounders including sex, a history of depression, cognitive functioning (ACE-R) and physical disability and handicap were analysed for inclusion in the image analysis, sex was the only variable significantly related to $\operatorname{mood}(p<0.0 .5)$. When the infarct analysis was repeated with gender as a covariate, infarct location was not significantly related to depression after a FDR correction was applied $(p>0.05)$. Additionally, when the infarct analysis was repeated excluding cases with no identifiable lesions on their MRI scan, infarct location was not significantly related to depression after a FDR correction was applied $(p>0.05)$. An independent samples t test indicated no significant difference in lesion volume between groups with and without depression $(\mathrm{p}>0.05)$.

\section{DISCUSSION}

Based on the results of this study, the contribution of lesion location to the aetiology of PSD remains inconclusive. In the planning of this study, we had followed the suggestions of previous investigators with regards to 
Table 1 Clinical characteristics of participants, $(\mathrm{N}=55)$

\begin{tabular}{|c|c|}
\hline Characteristic & \\
\hline Mean age $\pm S D$ (years) & $63(14)$ \\
\hline Range & $29-85$ \\
\hline Sex, n (\%) & \\
\hline Male & $31(56)$ \\
\hline Female & $24(44)$ \\
\hline Type of stroke, $n(\%)^{\star}$ & \\
\hline Ischaemic & $47(86)$ \\
\hline Haemorrhagic & $6(11)$ \\
\hline Laterality of stroke, $n(\%) \dagger$ & \\
\hline Left & $29(53)$ \\
\hline Right & $20(36)$ \\
\hline Bilateral & $5(9)$ \\
\hline Modified Rankin at Time 1†, n (\%) & \\
\hline 0-2 (no symptoms-slight disability) & $31(57)$ \\
\hline 3-5 (moderate-severe disability) & $23(43)$ \\
\hline Mean NIHSS at Time $1 \pm$ SD & $4 \pm 4$ \\
\hline Range & $0-21$ \\
\hline Mean ACE-R at Time $1 \pm S D$ & $78 \pm 16$ \\
\hline Range & $18-99$ \\
\hline Mean infarct volume $\pm S D \mathrm{~mL}$ & $19 \pm 53$ \\
\hline Range & $0-350 \ddagger$ \\
\hline English first language, $\mathrm{n}(\%)$ & $40(73)$ \\
\hline Personal history of depression, $\mathrm{n}(\%)$ & $12(22)$ \\
\hline Family history of depression, $\mathrm{n}(\%)$ & $5(9)$ \\
\hline Antidepressants during study, n (\%) & $3(6)$ \\
\hline Depression at Time 2, n (\%) & $15(27)$ \\
\hline
\end{tabular}

including stroke from all arterial territories but in doing so had unintentionally created challenges for ourselves. ${ }^{22}$ This study highlights the challenge in conducting voxel-based analysis for lesion location studies in PSD. These issues related primarily to the use of strict

Table 2 Frequency of depressed and non-depressed patients by infarct location identified on MRI scans

\begin{tabular}{|c|c|c|}
\hline \multirow[b]{2}{*}{ Infarct location } & \multicolumn{2}{|l|}{ Group } \\
\hline & Depressed & $\begin{array}{l}\text { Not } \\
\text { depressed }\end{array}$ \\
\hline Left hemisphere, n (\%) & $7(13)$ & $20(36)$ \\
\hline Left anterior, n (\%) & $4(7)$ & $13(24)$ \\
\hline Left posterior, n (\%) & $3(5)$ & $7(13)$ \\
\hline Right hemisphere, n (\%) & $5(9)$ & $15(27)$ \\
\hline Right anterior, n (\%) & $4(7)$ & $11(20)$ \\
\hline Right posterior, n (\%) & $1(2)$ & $3(5)$ \\
\hline $\begin{array}{l}\text { Extended across the } \\
\text { anterior and posterior } \\
\text { cortex, } \mathrm{n}(\%)\end{array}$ & 0 & $1(2)$ \\
\hline Bilateral, n (\%) & $1(2)$ & $2(4)$ \\
\hline No infarct on MRI scan & $2(4)$ & $2(4)$ \\
\hline
\end{tabular}

inclusion and exclusion criterion, principally, the exclusion of patients with severe dysphasia and patients who were likely to require institutionalised care. This action posed major challenges for voxel-based analysis which required the presence of regions which overlap with each other. Below we discus how these results compare with prior studies, the theoretical implications, the issues affecting our results and propose methods to overcome these shortcomings in future studies of PSD.

\section{Literature review}

The current findings differ from prior studies, many of which have proposed that lesions to the left frontal cortex and basal ganglia are related to the onset of PSD, and proximity of the lesion to the frontal pole is associated with severity of depression. ${ }^{8}{ }^{23-27}$ More recently, MRI studies have supplemented earlier CT studies, providing further support for a relationship between lesions to the basal ganglia and PSD. ${ }^{28}$ Other studies, however, have reported no significant relationship between lesion location and depression, with inconsistent findings attributed to methodological differences including timing of assessment. ${ }^{23}$ 29 Prior studies have defined lesion location based on whether lesions fall within a particular a priori defined region of the brain. ${ }^{8}$ Hence, while the results were less precise than voxel-based morphology, overlap of precise lesion location was not required. However, in the current study when the occurrence of depression was compared in patients with anterior and posterior strokes in left and right hemispheres, no relationship was found.

\section{Methodological considerations \\ Image analysis}

When analysing the findings of voxel-based analysis, one is often drawn to the significant results. A more useful approach is to examine the average map of all infarcts (figure 1) to ensure there are no regions without infarcts. In our case, the map showed a predilection of larger infarcts in the right hemisphere compared with the left. This visual analysis paralleled our finding with the infarct volume between the hemispheres. These findings preclude any further voxel-based analysis.

\section{Inclusion and exclusion criterion}

In our patients the infarct volumes were small as was the mean NIHSS score. This suggested that we had unintentionally recruited patients with mild strokes. This has occurred because of the concern that assessment of depression was unreliable in patients with severe dysphasia. The second problem occurred because patients with larger strokes had impaired alertness and awareness and were not able to provide informed consent; in this study we did not use carer consent as is often carried out in the initial stage of acute stroke trials. As was the case with our study, the results of prior works were likely biased due to the same pitfall of excluding patients with severe dysphasia. 


\section{Sample size}

One approach is to significantly increase the sample size to allow the chance of overlapping regions of infarct. However, a threefold increase in sample size would only result in an overlap of six scans. Hence, it is probable that the selection of 'minor' strokes is significantly contributing to this methodological issue. A larger sample size would not resolve this issue unless the inclusion and exclusion criteria are changed to prevent exclusion of aphasic and severe patients with stroke.

Strategies for recruitment may include assessment of patients in the subacute rather than the acute phase of stroke (Time 1). This may allow for some degree of neurological recovery, especially among patients with severe strokes, to increase the chance of recruitment of these patients. Alternatively, a non-verbal assessment of depression could be administered, such as the observerrated Stroke Aphasic Depression Quesionnaire- $10^{30}$ or the Aphasia Depression Rating Scale ${ }^{31}$; however, there is currently limited information regarding the psychometric properties of these tools which implies a validation study is necessary as a first step. Finally, restricting inclusion to patients with infarcts within an a priori defined anatomical region would increase overlap of infarcts, although this method has received criticism. ${ }^{22}$ Restricting arterial territory has previously lead to adequate overlap and significant results using voxelbased analyses. $^{9}$

\section{Future directions}

Despite the aforementioned challenges encountered with neuroimaging within this patient group, further research is warranted. A greater understanding of the lesion characteristics associated with PSD could lead to advances in diagnosis, preventive interventions and treatment. It is clear that PSD has an unfavourable influence on a range of outcomes; hence there is an important role for interventions. For preventive strategies to be implemented effectively, it is important to identify patients at greatest risk and to intervene, ideally within the acute poststroke period.

Voxel-based analysis could be used in future studies of comparable sample size if inclusion criteria were restricted to an a priori defined homogeneous subgroup of patients. This would allow hypothesis testing of the role of particular anatomical locations in PSD such as the basal ganglia. However, voxel-based analysis has limitations, as other potentially relevant infarct locations would not be detected using this approach. Future research should also take into account the influence of pre-existing lesions given the evidence to suggest that cumulative pre-existing lesions may be more important than the location of a single infarct. ${ }^{32}$

The high prevalence of depression found within this sample despite no particular significant lesion location related to depression points to the benefit of analysing networks of neural structures implicated in depression, rather than limiting the focus to the significance of lesions to one or two structures. An alternative method of analysis such as partial least-squares regression might therefore be more appropriate as this technique would allow the involvement of networks of neural structures to be explored. ${ }^{9}$ However, partial least-squares regression also relies on overlap of infarcted areas across MRI scans, a condition that was not met in the current data set. Further overlap of infarcts across scans could potentially be acquired if a non-verbal measure of depression was used to allow for the inclusion of patients with more severe strokes.

\section{CONCLUSIONS}

In summary, the results of this study do not provide evidence that infarcts to a particular neuroanatomical region contributes to PSD; however, results should be interpreted with caution due to methodological limitations. Future studies could overcome the methodological issues encountered during our study by assessing patients within the subacute rather than the acute poststroke period, using an observer-rated scale of depression rather than measures that require language comprehension abilities, or including patients with infarcts within an a priori defined anatomical region. Alternatively, an analysis of lesions to neurochemical networks implicated in depression could shed light on the debate.

Acknowledgements The authors sincerely thank the patients of the Stroke Units at Southern Health for their involvement in this study. The authors thank the Stroke Team for their support, in particular Mr David Ramsay for assisting with recruitment. The authors also thank Ms Imogen Rehm, Ms Kitty Wong and Ms May Chong who contributed to data collection.

Contributors SAG designed the study, recruited patients, collected, analysed data, drafted and revised the paper. TGP assisted with the study design, monitored data collection, segmented the infarcts on the MRI scans, assisted with image analysis and critically reviewed the paper. AGW assisted with the study design, monitored data collection, assisted with image analysis and critically reviewed the paper. JC assisted with image analysis and critically reviewed the paper. KV assisted with the study design and critically reviewed the paper.

Funding This research received no specific grant from any funding agency in the public, commercial or not-for-profit sectors.

\section{Competing interests None.}

Ethics approval Southern Health and Monash University Human Research Ethics Committees.

Provenance and peer review Not commissioned; externally peer reviewed.

Data sharing statement No additional data are available.

Open Access This is an Open Access article distributed in accordance with the Creative Commons Attribution Non Commercial (CC BY-NC 3.0) license, which permits others to distribute, remix, adapt, build upon this work noncommercially, and license their derivative works on different terms, provided the original work is properly cited and the use is non-commercial. See: http:// creativecommons.org/licenses/by-nc/3.0/

\section{REFERENCES}

1. Murata Y, Kimura M, Robinson RG. Does cognitive impairment cause poststroke depression. Am J Geriatr Psychiatry 2000;8:310.

2. Gainotti G, Antonucci G, Marra C, et al. Relation between depression after stroke, antidepressant therapy, and functional recovery. J Neurol Neurosurg Psychiatry 2001;71:258-61. 
3. Chemerinski E, Robinson RG, Kosier JT. Improved recovery in activities of daily living associated with remission of poststroke depression. Stroke 2001;32:113-17.

4. Kang HJ, Stewart R, Park M-S, et al. White matter hyperintensities and functional outcomes at 2 weeks and 1 year after stroke. Cerebrovasc Dis 2013;35:138-45

5. Suenkeler IH, Nowak M, Misselwitz B, et al. Timecourse of health-related quality of life as determined 3, 6, and 12 months after stroke. Relationship to neurological deficit, disability and depression. J Neurol 2002;249:1160-7.

6. Hackett ML, Anderson CS. Predictors of depression after stroke: a systematic review of observational studies. Stroke 2005;36:2296-301.

7. Robinson RG, Shoemaker WJ, Schlumpf M, et al. Effect of experimental cerebral infarction in rat brain on catecholamines and behaviour. Nature 1975;255:332-4.

8. Robinson RG, Kubos KL, Starr LB, et al. Mood disorders in stroke patients: importance of location of lesion. Brain 1984;107:81-93.

9. Phan TG, Chen J, Donnan G, et al. Development of a new tool to correlate stroke outcome with infarct topography: a proof-of-concept study. Neuroimage 2010;49:127-33.

10. Carson AJ, MacHale SM, Allen K, et al. Depression after stroke and lesion location: a systematic review. Lancet 2000;356:122-6.

11. Singh A, Hermann N, Black SE. The importance of lesion location in poststroke depression: a critical review. Can J Psychiatry 1998;43:921-7.

12. Benton AL, Hamsher Kd, Sivan AB. Multilingual aphasia examination. 3rd edn. lowa City: Psychological Assessment Resources, 1994.

13. Mioshi E, Dawson K, Mitchell J, et al. The Addenbrooke's Cognitive Examination Revised (ACE-R): a brief cognitive test battery for dementia screening. Int J Geriatr Psychiatry 2006;21:1078-85

14. Lyden $\mathrm{P}$, Lu M, Jackson $\mathrm{C}$, et al. Underlying structure of the national institutes of health stroke scale: results of a factor analysis. NINDS tPA Stroke Trial Investigators. Stroke 1999;30:2347-54

15. Rankin J. Cerebral vascular accidents in patients over 60 , ii: prognosis. Scott Med J 1957;2:200-15.

16. van Swieten J, Koudstaal $P$, Visser $M$, et al. Interobserver agreement for the assessment of handicap in stroke patients. Stroke 1988;19:604-7.

17. Zigmond AS, Snaith RP. The Hospital Anxiety and Depression Scale. Acta Psychiatr Scand 1983;67:361-70.
18. Sheehan DV, Lecrubier $\mathrm{Y}$, Harnett-Sheehan $\mathrm{K}$, et al. Reliability and validity of the Mini International Neuropsychiatric Interview (M.I.N.I.) according to the SCID-P. Eur Psychiatry 1997;12:232-41.

19. APA. Diagnostic and statistical manual of mental disorders. 4th edn. Text Revision (DSM-IV-TR). Arlington: American Psychiatric Association, 2000.

20. Aben I, Verhey FRJ, Honig A, et al. Research into the specificity of depression after stroke: a review on an unresolved issue. Prog Neuropsychopharmacol Biol Psychiatry 2001;25:671-89.

21. Genovese CR, Lazar NA, Nichols T. Thresholding of statistical maps in functional neuroimaging using the false discovery rate. Neuroimage 2002;15:870-8.

22. Hand PJ, Wardlaw JM, Rivers CS, et al. MR diffusion-weighted imaging and outcome prediction after ischemic stroke. Neurology 2006;66:1159-63.

23. Narushima K, Kosier JT, Robinson RG. A reapprasial of poststroke depression, intra- and inter- hemispheric lesion location using meta-analysis. J Neuropsychiatry Clin Neurosci 2003;15:422-30.

24. Lipsey JR, Robinson RG, Pearlson GD, et al. Mood change following bilateral hemisphere brain injury. $\mathrm{Br} J$ Psychiatry 1983;143:266-73.

25. Morris PL, Robinson RG, Raphael B, et al. Lesion location and poststroke depression. J Neuropsychiatry Clin Neurosci 1996;8:399-403.

26. Robinson RG, Price TR. Post-stroke depressive disorders: a follow-up study of 103 outpatients. Stroke 1982;13:635-41.

27. Starkstein SE, Robinson RG, Price TR. Comparison of cortical and subcortical lesions in the production of poststroke mood disorders. Brain 1987;110:1045-59.

28. Vataja R, Leppavuori A, Pohjasvaara T, et al. Poststroke depression and lesion location revisited. J Neuropsychiatry Clin Neurosci 2004;16:156-62.

29. Bhogal SK, Teasell R, Foley N, et al. Lesion location and poststroke depression: systematic review of the methodological limitations in the literature. Stroke 2004;35:794-802.

30. Sutcliffe LM, Lincoln NB. The assessment of depression in aphasic stroke patients: the development of the Stroke Aphasic Depression Questionnaire. Clin Rehabil 1998;12:506-13.

31. Benaim C, Cailly B, Perennou D, et al. Validation of the aphasic depression rating scale. Stroke 2004;35:1692-6.

32. Santos M, Gold G, Kövari E, et al. Differential impact of lacunes and microvascular lesions on poststroke depression. Stroke 2009;40:3557-62. 